\title{
FOUR METRIC CONDITIONS CHARACTERIZING ČECH DIMENSION ZERO
}

\author{
KEVIN BROUGHAN
}

\begin{abstract}
If $(X, d)$ is a metric space let $d_{x}(y)=d(x, y)$. It is proved that if each $x$ in $X$ has a neighbourhood $P$ with $d_{x}(P)$ not dense in any neighbourhood of 0 in $[0, \infty)$ then Ind $X=0$. This metric condition characterizes metrizable spaces which have $\check{C}$ ech dimension zero. Three other metric characterizations are given.
\end{abstract}

Introduction. In [1], [2] and [3] metric conditions sufficient for a metrizable topological space to have Čech dimension zero are given. In [3, Theorem 3.4, p. 66] it is shown that if a space $X$ has a compatible metric $d$ with range not dense in any neighbourhood of 0 in $[0, \infty)$ then Ind $X=0$. In this paper it is shown that the conclusion is the same when the condition on the metric holds only "locally" or "pointwise". Specifically, if $d$ satisfies for each $x$ in $X$ there is a neighbourhood $P$ of $x$ such that $d_{x}(P)$ is not dense in any neighbourhood of 0 in $[0, \infty)$ then Ind $X=0$. Conversely, if a metrizable space has Čech dimension zero then there exists a compatible metric satisfying the given condition globally and hence locally. Three other related characterizations are given.

First two preliminary constructions are made. Let $(X, d)$ be a metric space. If $A \subset X$ is not empty let $d_{A}(x)=\inf \{d(a, x): a \in A\}$ for each $x$ in $X$. Let $d_{x}=d_{\{x\}}$. For each $n$ in $\mathbf{N}$ let

$$
E(n)=\left\{x \in X: d_{x}(X) \text { is not dense in }\left[2^{-n-1}, 2^{-n}\right]\right\} .
$$

Then each $E(n)$ is open.

For each pair of rational numbers $r$ and $s$ with $0<r<s$ let

$$
A(r, s)=\left\{x \in X: d_{x}(X) \cap[r, s]=\varnothing\right\} .
$$

For each rational $\varepsilon$ with $r<\varepsilon<s$ let

$$
\mathscr{B}(r, \varepsilon, s)=\{B(x, \varepsilon): x \in A(r, s)\}
$$

where $B(x, \varepsilon)$ is the metric ball of center $x$ and radius $\varepsilon$. The family $\mathscr{B}(r, \varepsilon, s)$ is closure preserving and consists of sets which are closed and open. Well $\operatorname{order} A(r, s)$ and, for each $x$ in $A(r, s)$ and $\varepsilon$ in $\mathbf{Q}$ with $r<\varepsilon<s$, let

Received by the editors November 30, 1976.

AMS (MOS) subject classifications (1970). Primary 54E35, 54E45.

Key words and phrases. Metric spaces, Cech dimension zero.

(1) American Mathematical Society 1977 


$$
D(x, \varepsilon)=B(x, \varepsilon) \backslash \bigcup_{y<x} B(y, \varepsilon) .
$$

Let

$$
\mathscr{D}(r, \varepsilon, s)=\{D(x, \varepsilon): x \in A(r, s)\} .
$$

The family $\mathscr{D}(r, \varepsilon, s)$ is closure preserving and consists of disjoint closed and open sets. Finally let

$$
\mathscr{B}=\{\mathscr{D}(r, \varepsilon, s): 0<r<\varepsilon<s \text { and } r, \varepsilon, s \in \mathbf{Q}\} .
$$

Then $\mathscr{B}$ is a $\sigma$-locally finite family of subsets of $X$ which are closed and open.

TheOREM 1. Let $(X, d)$ be such that each $x$ in $X$ has a neighbourhood $P$ satisfying $d_{x}(P)$ is not dense in any neighbourhood of 0 in $[0, \infty)$. Then Ind $X=0$.

Proof. Under the given conditions the family $\mathscr{B}$ is a $\sigma$-locally finite base for $X$ consisting of sets which are closed and open. Thus, by [4, Theorem 5, p. 291], Ind $X=0$.

The hypothesis of Theorem 1 may be restated as follows: each $x$ in $X$ is in $E(n)$ for an infinite number of integers $n$ in $\mathbf{N}$.

THEOREM 2. Let $E(n)$ be dense in $X$ for an infinite number of positive integers $n$. Then Ind $X=0$.

Proof. The proof consists in showing that $\mathscr{B}$ is a base for $X$. Let $P$ be open and let $x \in P$. Let $n$ in $\mathbf{N}$ satisfy $B\left(x, 2^{-n+1}\right) \subset P$ and $E(n)$ is dense in $X$. Let $y \in E(n) \cap B\left(x, 2^{-n-1}\right)$. Then there exist rational numbers $r, s, \varepsilon$, with $2^{-n-1}<r<\varepsilon<s<2^{-n}$ and such that $y \in A(r, s)$. Because $x \in B(y, \varepsilon)$ it follows that there is a $z$ in $A(r, s)$ with $x \in D(z, \varepsilon) \subset B(y, \varepsilon) \subset B\left(x, 2^{-n+1}\right)$ $\subset P$. This completes the proof.

TheOREM 3. If $d_{F}(X)$ is not a neighbourhood of 0 in $[0, \infty)$ for all closed subsets $F$ in $X$ then Ind $X=0$.

Proof. Let $P \subset X$ be any open subset. Then there exists a sequence $a_{n} \downarrow 0$ in $[0, \infty)$ such that $a_{n} \notin d_{X-P}(X)$ for each $n$ in $\mathbf{N}$.

Let $G_{i}=\left\{x \in X: d_{X-P}(x)>a_{i}\right\}$. Then $P=G_{1} \cup\left(G_{2} \backslash G_{1}\right) \cup\left(G_{3} \backslash G_{2}\right)$ $\cup \ldots$ The sets in this union are closed, open and disjoint. Since every open set has such a decomposition it follows from [4, Theorem 5, p. 291] that Ind $X=0$.

TheOREM 4. Let $(X, d)$ be a metric space and suppose that there exists a sequence $\left(d_{n}\right)$ of continuous metrics on $X$ converging uniformly to $d$ and such that each $d_{n}$ satisfies either the hypothesis of Theorem 1 or of Theorem 2 . Then Ind $X=0$.

Proof. Let $X_{n}=\left(X, d_{n}\right)$ and consider the mapping 


$$
f: X \rightarrow \prod_{n=1}^{\infty} X_{n}=Y
$$

defined by $x \rightarrow(x, x, x, \ldots)$. The map $f$ is an embedding. By Theorems 1 and 2 , for each $n$, Ind $X_{n}=0$. Hence Ind $Y=0$. Therefore Ind $X=0$ since $Y$ is metrizable. This completes the proof.

\section{REFERENCES}

1. K. A. Broughan, A metric characterizing $\check{C}$ ech dimension zero, Proc. Amer. Math. Soc. 39 (1973), 437-440.

2. , Metrization of spaces having Čech dimension zero, Bull. Austral. Math. Soc. 9 (1973), 161-168.

3. Invariants for real-generated uniform topological and algebraic categories, Lecture Notes in Math., vol. 491, Springer-Verlag, Berlin and New York, 1975.

4. R. Engelking, Outline of general topology, North-Holland, Amsterdam, 1968.

Department of Mathematics, University of Waikato, Hamlton, New Zealand 